International Journal on Information Theory (IJIT), Vol.3, No.1, January 2014

\title{
OPTIMIZED Routing AND PIN-CONSTRAINED DESIGN OF DIGITAL MICRO-FLUIDIC BIOCHIP
}

\author{
Nabonita Das ${ }^{1}$, Susanta Chakraborty ${ }^{2}$ and Parthasarathi Dasgupta ${ }^{3}$ \\ ${ }^{1}$ Bengal Engineering and Science University, Shibpur, India \\ ${ }^{2}$ Bengal Engineering and Science University, Shibpur, India \\ ${ }^{3}$ Indian Institute of Management Calcutta, India
}

\begin{abstract}
Digital micro-fluidic biochips have represented as a small integrated tool for large biological sample analysis. Only nanoliter volume of discrete fluid droplets (sample) is required to manipulate the integrated chips on an electrode array via electrical actuation. Each electrode activate with independent pin for direct addressing biochip. For low cost and disposal biochip, pin-constraint design is one of the main motivations of this paper. However the pin-count reductions are inescapably depend on the droplet routing stage. The emphasis here is on the concurrent routing with minimum number of cell used without any electrode interference. The paper presents a multi-objective optimization technique for concurrent routing on single source -single target net (2-pin net), two-source single target net (3-pin net) problem and integrates the routing result with masking based algorithm to select the compatible sequence without any electrode interference. The experimental result of benchmark Invitro, Protein show the significant reduction of control pins, number of used cells and routing time compare to crossreferencing and broadcast addressing, ant colony optimization and two-stage ILP method.
\end{abstract}

\section{KEYWORDS}

Micro-fluidic Biochip, multi-objective optimization, droplet routing, pin-constrained design.

\section{INTRODUCTION}

Micro-fluidic Biochips constitutes an emerging technology area that opens up wide range of versatile research area. The basic device operation is based on micro liter and nano-liter volumes of fluid (droplet) containing biological samples on a two dimensional electrode array, utilizing the phenomenon of electro-wetting to manipulate and move the droplets in desired direction. A digital micro fluidic biochip consists of a two-dimensional electrode array. Two parallel plates consist for a unit cell of the electrode array. A patterned array of electrodes contains at the bottom plate, and a continuous ground electrode is coated at the top plate. Pin constrained digital micro-fluidic biochips, one of the major genres of the pin-count reduction approaches, reduce the number of pins to be routed to the electrodes by assigning each control pin to multiple electrodes; that is, multiple electrodes are controlled by a single control signal, and thus they are turned on/off simultaneously. Now droplet routing is the one of the important

DOI : $10.5121 /$ ijit.2014.3103 
issue in design of PDMFB, where multiple droplet share same pin over the microarray. Routing information is very much correlated with pin-constraint design.

In this paper we propose a multi objective optimization technique based on Particle Swarm Optimization (PSO) concept to find a solution of droplet routing in DMFB. However, PSO aims to obtain the optimal solution instead of the searching path, while the purpose of our work concentrates on the control of the crowd (multiple net) movement, which is composed of the generated searching paths of particles. Here, we focus on finding non-overlapping, smoothing path for each net. Next we design pin-constrained design using the routing result. The proposed method for pin- constrained design based on the masking base technique, combining the compatible control sequence with the same pin. We evaluate the proposed method on Invitro, Protein and Test benchmark. The rest of the paper follows as, section2 provides with an overview of the major works in this area. Section3 describes formulation of particle swarm optimization for droplet routing. Our proposed method is described in section 4 followed by experimental results in section5. Finally section6 concludes the work with possible future directives.

\section{RELATED WORKS}

Micro-fluidic technology is recently emerged into several field of application and Digital Microfluidic Biochip is a renowned in venture of fluidic technology. Digital Micro-fluidic technology is the efficient for parallel processing, reconfiguration and resource utilization. So, researchers are mainly focused for more customized design for Biochip.

There are many methods to solve droplet routing problem. One is the prioritized $\mathrm{A}^{*}$-search algorithm [5], each droplet is assigned a priority, and the A-search algorithm is used to coordinate each droplet based on its priority. The drawback of this approach is that they did not consider the practical timing constraint for throughput consideration. They only considered two-pin net. Another approach based on open shortest path first routing protocol [6]. A global moving vector based analysis construct a convincing routing path to minimize the number of electrodes. They proposed an entropy [7] based mechanism to schedule the nets and then compaction technique using dynamic programming to minimize the latest arrival time. A fault tolerant routing mechanism is proposed in [8]. Another concept of cross-router [9] is used a weighted maze routing framework to move an amount of droplets in a parallel fashion to optimize the resource utilization. In [10] a network flow based two stage method has been proposed. Here, an undirected graph considering the source and destination pair and then perform a high level routing based on "Minimum Cost and Maximum Flow" technique. However final scheduling is not possible through high level routing because the dependency graph was prepared by only considering the fluidic constraint. Most well known techniques Progressive Integer Linear Programming (ILP) based algorithm has been proposed in [11].An contamination

issues in Digital Micro-fluidic Biochip due to bio-molecular residues represented in [12].A meta-heuristic strategy based on Ant-colony-optimization (ACO) proposed in [13].Our method is also a meta-heuristic optimization method but we consider the collision avoidance concurrent routing with pin count reduction. Pin-constrained design of digital Micro-fluidic biochips was proposed in [14]. This method uses array partitioning and careful pin assignment 
International Journal on Information Theory (IJIT), Vol.3, No.1, January 2014

to reduce the number of control pins. An alternative method based on a cross reference driving scheme is presented in [15]. This method allows control of an $N \times M$ grid array with only $N+M$ control pins, but due to electrode interference, this design cannot handle the simultaneous movement of more than two droplets. Broadcast addressing method [16] and ILP based [17] method also significantly reduce the number of control pin but methods are not totally interference free. Our method is optimized the routing time and used cell and pin assignment.

\section{Problem Formulations}

Droplet routing is the important step for scheduling the bioassay in physical modeling. A routing sub problem includes the routing path and specifies the location where the droplet stays along the corresponding routing path at specific clock cycle. In a specific time instance, there can be multiple droplets moved into their target location simultaneously. So, they can be adjacent to each other at any movement and cause an unwanted mixing. Droplet routing algorithm is maintained a terminology that no two droplets are adjacent at a same time instance. The Droplets are moved either horizontally (row-wise) or vertically (column-wise).At the time of routing, they always maintain some fluidic constraints (static or dynamic)[16]. Each net should be routed in between the upper bound of droplet routing time.

The droplet routing information is stored in the form of electrode-actuation sequence, where each bit represents the status of electrode at particular time step. The status can be either " 1 " (activate), "0" (deactivate), or "x" (don't-care). At every time step, the sequence of two electrode activation sequence is compatible if the value of two bits are same or substituting the ' $x$ ' bit with 1 or 0 bit. A group of electrodes whose electrode-actuation sequences are mutually compatible can be controlled using the same pin. That means the same number of control pin use in many electrodes over the microarray.

The optimization problem for droplet routing and pin assignment can be stated as follows.

Input: For each net source and target pair coordinate value and blockage position.

Output: Optimal routing path for each sub problem and a mapping of control pins to electrodes for droplet routing in all sub problems without any pin-actuation conflict.

\subsection{Multi-objective Optimization}

The primary objective of droplet routing is to find the shortest path between source- target pair and minimize the used cells. This droplet routing problem can be viewed as a multi-objective optimization problem, where some of the constraints are optimized simultaneously. Concurrent movement of droplets over the chip area efficiently uses the resources and makes the operations.

In this paper, PSO (particle swarm optimization) heuristic is explored that has been inspired by swarm intelligence. The principal operation of PSO is to optimize a problem by having a population of candidate solutions that represents as particles. Every particle adopts its position exchanging the information from neighborhood particle position. In our problem, we represent particle as each net routing path for in between source and target pair. Here, each particle must avoid the collision with static obstacles. Static obstacles are waste reservoirs or on chip reservoirs that occupy some of the electrode on DMFB chip. Each particles position act as a dynamic 
International Journal on Information Theory (IJIT), Vol.3, No.1, January 2014

obstacle with respect to other particles. Dynamic obstacles overcome with maintaining fluidic constraints.

\subsection{Fluidic Constrained}

There have an important constraint [16] which are significant in case of routing as well as in pin constrained design. The fluidic constraints are used to avoid unexpected mixtures between two droplets of different nets during their transportation and it can be further divided into the static fluidic constraint and dynamic fluidic constraint.

Let di at (x it, y it ) and dj at (x jt, y jt) denote two independent droplets at time t.

Then, the following constraints should be satisfied for any $t$ during routing: Then, the following constraints should be satisfied for any t during routing:

Static constraint:

$$
\left|\mathrm{x}^{\mathrm{i}}{ }_{\mathrm{t}}-\mathrm{x}_{\mathrm{t}}^{\mathrm{j}}\right|>1 \text { or }\left|\mathrm{y}_{\mathrm{t}}^{\mathrm{i}}-\mathrm{y}_{\mathrm{t}}^{\mathrm{j}}\right|>1
$$

Dynamic constraint:

$$
\begin{aligned}
& \left|\mathrm{x}^{\mathrm{i}}{ }_{\mathrm{t}+1}-\mathrm{x}^{\mathrm{j}}{ }_{\mathrm{t}}\right|>1 \text { or }\left|\mathrm{y}^{\mathrm{i}}{ }_{\mathrm{t}+1}-\mathrm{y}^{\mathrm{j}}{ }_{\mathrm{t}}\right|>1 \text { or }\left|\mathrm{x}^{\mathrm{i}}{ }_{\mathrm{t}}-\mathrm{x}^{\mathrm{j}}{ }_{\mathrm{t}+1}\right|>1 \text { or } \\
& \left|\mathrm{y}^{\mathrm{i}}{ }_{\mathrm{t}}-\mathrm{y}^{\mathrm{j}}{ }_{\mathrm{t}+1}\right|>1
\end{aligned}
$$

The static fluidic constraint signifies that the minimum spacing between two droplets is one cell for any time $t$ during routing. The dynamic Fluidic constraint species that the activated cell for di cannot be adjacent dj between successive time cycles $t$ and $t+1$. Otherwise, there can be more than one activated neighboring cell for $\mathrm{dj}$, which may lead to errant fluidic operations.

\subsection{Electrode Constraint}

If a droplet is located at cell (x,y) at time $t$, this cell $(x, y)$ must be activated. There would be total four cells, just neighbor of activated cell, must be deactivated. At each routing step, a droplet position create an electrode activation sequence of " 1 " (activate), " 0 " (deactivate), or " $x$ " (don't-care). This electrode activation sequence is used for pin reduction.

\section{Proposed Method}

Generally, Particle Swarm Optimization is only interested in the target position of particles but in our problem, we must care about particle path at each step. Here routing path in each net represent by one particle. Swarm search space is similar to the each of the net area. The routing paths for the droplets are always horizontal and vertical without any diagonal movement. The routing path represent as, we check the source coordinate with target coordinate and increase or decrease until equal to the target coordinate. At this time, we can't consider any information of blockage area. At the end of the initialization phase, every route path produces a fitness value. Fitness value is the objective function that satisfied the requirement. The fitness function is represented as, 
Fitness function $=\max \sum^{N_{i=1}}\left\{\operatorname{Cost}\left(s, o_{i}\right) / \operatorname{Cost}(s, t)\right\}$,

Here, $\mathrm{N}=$ total number of obstacle $\mathrm{o}_{\mathrm{i}}=\mathrm{i}^{\text {th }}$ Obstacle

Here, the fitness function is maximized the fitness value in each iteration. The function $\operatorname{cost}(\mathrm{s}, \mathrm{o})$ measure the distance between source and obstacle. The function $\operatorname{cost}(\mathrm{s}, \mathrm{t})$ measures the distance between source and target. Objective is to avoid the obstacle with minimum electrode. In the fitness function equation, the numerator value is greater than the denominator value; convey that droplet can be reached into target without obstacle. So, fitness value would be greater than one or any greater value. If any fitness value is not greater than one for one obstacle or greater than one but minimum value respect to all obstacles that means the routing path may be belonged into the obstacle. The routing path's coordinate change around the edge of the obstacle because that would be the shortest path avoiding obstacle. Every net routing path is the local best (pbest) and the routing path has highest fitness value that is the global best (gbest) among all pbest. If any pbest coordinate intersect with gbest coordinate at same timestamp or greater than or less than one time stamp, pbest coordinate change the value of coordinate with respect to intersecting position and target position. In this way, iteration continues until all net routes concurrently. We find the non-overlapping concurrent routing path for each net.

Then, we construct the undirected graph for each net. Now each vertex store the control-signal sequence of each control pin ,control signal sequence consists of the values 1(activated),0(deactivated), and X(don't care). Each incident edge represents the Masking sequence of vertex's control actuation sequence. Then we find the same Masking sequence from several edges and combine the non-adjacent vertex with same pin. This way, the several electrodes can be activated with same pin.

\subsection{Droplet Routing Algorithm}

A procedural form of our proposed algorithm is described below.

Input

1) Two-dimensional structure of microarray (total number of rows and columns)

2) Blocking coordinate for blocked electrode

3) Source - target pair $(\mathrm{S}, \mathrm{T})$

Output

1) Optical routing path for each $(S, T)$ pair.

Step 1: First create the routing path for each net into its bounding box. The method is adopted by the way, droplet moves only horizontally and vertically between the bounding boxes. The source and target y coordinate increase or decrease depending on the position until each $y$ coordinates are equal. Then check the x coordinate position, increase or decrease until source coordinate change into target coordinate. Now, total routing paths of each net represent as a particle. 
Step 2: Fitness value is the objective function that satisfied our requirement. Here, the fitness function is calculated as,

$$
\begin{aligned}
& \text { Fitness value }=\max \sum^{N_{i}=1}\{\operatorname{Cost}(s, o i) / \operatorname{Cost}(s, t)\} \\
& \text { Here, } N=\text { total number of obstacle } \\
& \text { oi }=i^{\text {th }} \text { Obstacle } \\
& \operatorname{Cost}(s, o)=\left(s_{x}-o_{x}\right)^{2}+\left(s_{y}-o_{y}\right)^{2} \\
& \operatorname{Cost}(s, t)=\left(s_{x}-t_{x}\right)^{2}+\left(s_{y}-t_{y}\right)^{2}
\end{aligned}
$$

The function $\operatorname{cost}(\mathrm{s}, \mathrm{o})$ measure the distance between source and obstacle. The function cost $(\mathrm{s}$, t) measure the distance between source and target. Our objective is to avoid the obstacle with minimum electrode. In the following equation, when the numerator value is greater than the denominator value, convey that droplet can be reached into target without obstacle. So, fitness value would be greater than one. If any of the particle fitness value is not greater than one or minimum value respect to all obstacles, require checking any coordinate of routing path belonging to the obstacle. Let, $\mathrm{V}=\{\mathrm{Vk} 1, \mathrm{Vk} 2, \ldots ., \mathrm{Vkk}\}$, it is the set of vertex of shortest route but some the vertex belong into the obstacle region. Then, the virtual vertex is selected $\left(\mathrm{V}^{\prime} € \mathrm{~V}\right)$, outside the boundary of obstacle.Step 3: Droplet must route around the edge of the obstacle. Vertex V (source vertex) generates a virtual vertex $V^{\prime}$, which has the same horizontal or vertical coordinate with the left (or right) edge of the obstacle with respect to position of the obstacle boundary and droplet position. The next is to connect V, V' and T sequentially as per rule of Step 1 .

Step 4: The fitness function of any particle (net), consider all obstacle respect to the droplet source position and value is greater than one, that routing path is local optimum (pbest) of the net. If any routing path fell under Step 3 condition, then new shortest path $\left(L\left(V, V^{\prime}\right)+L\left(V^{\prime}, T\right)\right)$ is used to update the route path of that net.

Step 5: Then, the gbest is found from all pbest that has the highest fitness value because that route path has lower probability to go through the obstacle and cover the minimum electrode to reach in target.

Step 6: Now, intersection position of any pbest is updated with comparison of gbest. If gbest route path shares the common vertex with other pbest (other routing path), the pbest droplet's route path is used this equation for changing the position,

$$
\mathrm{D}_{\mathrm{i}}{ }^{\mathrm{k}+1}=\mathrm{D}_{\mathrm{i}}^{\mathrm{k}}+\mathrm{c} 1 * \mathrm{r} 1 *\left(\text { pbest }_{\mathrm{i}}{ }^{\mathrm{k}}-\mathrm{S}_{\mathrm{i}}\right)+\mathrm{c} 2 * \mathrm{r} 2 *\left(\text { gbest }_{\mathrm{i}}-\mathrm{S}_{\mathrm{i}}\right)
$$

where $\mathrm{k}$ denotes the $\mathrm{k}$-th generation. Both $\mathrm{c} 1$ and $\mathrm{c} 2$ are constant real positive number. The $\mathrm{r} 1$ and $\mathrm{r} 2$ are random real numbers belonging to [0,0.1]. Here, pbesti is target coordinate of pbest (other routing path) and gbest is the target coordinate of gbest, $\mathrm{S}_{\mathrm{i}}$ is the conflicting position of pbest and gbest. 
Droplet position is updated by equation,

$$
\mathrm{Si}=\mathrm{Si}+\mathrm{Di}
$$

When, the position of conflicting coordinate is changed, the algorithm is concerned about that the change coordinate value is always in between source and target coordinate of its own net (in between bounding box). The pbest coordinate changes, and it is the new position, again step 1 to step 5 repeat.

Step 7: Iteration continue until all net satisfies concurrent routing without any violation of fluidic constraint or any conflict. The gbest is calculated from the rest of the pbest because current iteration gbest complete its execution.

\subsection{3-Pin net routing}

Many multi-functional biochip consist with three -pin net (multi-pin) because several on-chip operations are carried on. A three-pin net has two source locations. Biological samples from these two source locations move towards a same target location. On their way moving towards the target there is a common point called mixing point, where the two droplets get merged, then choose a single path to route to the target, and in their way towards the target location droplets get mixed. The three-pin net problem can be acted as two 2-pin net problems.

Three-pin net can be described as two source droplet and one target location. Two droplets try to merge at the mixing position, then act as single droplet and go to the target location.

Step1: Find the fitness value for each droplet of 3-pin net taking mixing position as a target.

Step2: The lowest fitness value droplet start first rather than other one because two droplets are required in between one distance in the place of mixing position at the same timestamp. If fitness value is equal for both, they start at the same time.

Step3: Another droplet starts after the few timestamp that is the distance difference between the droplet's sources to target (mixing position). Then they act as a single droplet after the merging without losing any timestamp and go towards in the final target position as 2-pin net.

\subsection{Steps of Pin -assignment}

Step 1: From the routing algorithm, get the information about routing of net, i.e. approximately found how much electrode is used.

Step 2: Construct an undirected graph, as each electrode represent as a vertex and each horizontally and vertically adjacent electrode connect with edge because the electrode can only move horizontally and vertically.

Step 3: Now each vertex store the control-signal sequence of each control pin ,control signal sequence consists of the values 1 (activated), 0 (deactivated), and X(don't care).

Step 4: Each vertex stores the control sequence of that electrode, and each edges represent the masking sequence of vertexes of incident edge. When each bit of the both sequences are 1 's or any of the other is X's, the making bit is 1's. Each bit of the both sequences are 0's or any 
of the other is X's, the making bit is 0's. Each bit of the both sequences are X's or any of the other is 0 's, the making bit is X's.

Step 5: Now, find the compatible sequence that maintain all pin-constraint for any sequence. Choose any vertex (like V1), find out that electrode activation sequence of this vertex is derived from any other masking sequence (just change the don't care term ' $\mathrm{X}$ ' as 0 or 1).

Step 6: The algorithm is chosen masking sequence in such a way that any vertex of the incident edge cannot be adjacent with the choosing vertex.

Step 7: After selecting the masking sequence, select the vertex of the incidence edge that is the farthest from the choosing vertex to maintain control electrode constraint, $|x i-x j| \geq 2$ and $|y i-y j| \geq 2$.

Step 8: Compute the combined sequence.

Step 9: For each net, the algorithm is computed step1 to step9, then find the compatible sequence between the different nets combined sequence. Here also the algorithm is checked that no two vertexes of the different nets are adjacent to each other at same time stamp.

\subsubsection{Program code for Pin-assignment}

Each electrode $E_{i}$ activation sequence represent as $A C{ }_{i}=\left\{a_{1}^{i}, a_{2}^{i}, \ldots \ldots \ldots \ldots . . . a_{n}^{i}\right\}$

Assign $\quad w_{-}$Eij(weight of edge in between $\mathrm{Ei}$ and $\mathrm{Ej}$ ) =

Sequence of pin actuation of $\mathrm{Ei}$ OR sequence of pin actuation of $\mathrm{Ej}$

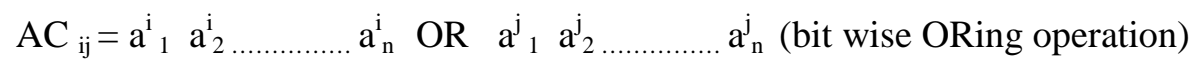

While ( $\mathrm{G}$ is not empty) do

For (Each electrode sequence $\mathrm{AC}_{\mathrm{i}}$ )

For (Each ORing sequence $\mathrm{AC}_{\mathrm{jk}}$ )

If $\mathrm{AC}_{\mathrm{i}}$ is equal to $\mathrm{AC}_{\mathrm{jk}}$ and $\mathrm{d}(\mathrm{i}, \mathrm{j})>\mathrm{d}(\mathrm{i}, \mathrm{k})$ then

Compute _ sequence $\left(\mathrm{AC}_{\mathrm{i}}, \mathrm{AC}_{\mathrm{j}}\right)$

set $\mathrm{AC}_{\mathrm{i}}<-$ Compute_sequence $\left(\mathrm{AC}_{\mathrm{i}}, \mathrm{AC}_{\mathrm{j}}\right)$

\section{End}

\section{End}

$\mathbf{C S}^{\mathrm{ij}}$ be the set combining the control sequence of $\mathrm{A} \mathbf{C i}, \mathrm{ACj}$

$$
\begin{gathered}
\text { Compute_sequence }\left(A C_{i}, A C_{j}\right) \\
A C_{i}=\left\{a^{i}{ }_{1}, a^{i} a_{2}, \ldots \ldots \ldots \ldots ., a_{n}\right\} \\
A C_{j}=\left\{a^{j}{ }_{1}, a_{2}^{j}, \ldots \ldots \ldots \ldots \ldots, a_{n}^{j}\right\}
\end{gathered}
$$


International Journal on Information Theory (IJIT), Vol.3, No.1, January 2014

For $(z=1 \ldots \ldots . n) / / z$ is $1 \ldots \ldots . . n$ (total sequence)

For (each $\mathrm{a}^{\mathrm{i}}{ }_{\mathrm{z}}$ and each $\mathrm{a}_{\mathrm{z}}^{\mathrm{j}}$ )

If $\left(\mathrm{a}_{\mathrm{z}}^{\mathrm{i}}=1\right.$ and $\left.\mathrm{a}_{\mathrm{z}}^{\mathrm{j}}=\mathrm{X}\right)$ or $\left(\mathrm{a}_{\mathrm{z}}^{\mathrm{i}} \mathrm{X}\right.$ and $\left.\mathrm{a}_{\mathrm{z}}^{\mathrm{j}}=1\right)$

$\mathrm{CS}^{\mathrm{ij}}{ }_{\mathrm{z}}=1$

Else if $\left(a^{i}{ }_{z}=0\right.$ and $\left.a^{j}=X\right)$ or $\left(a^{i}{ }_{z}=X\right.$ and $\left.a^{j}{ }_{z}=0\right)$ or $\left(a^{i}{ }_{z}=0\right.$ and $\left.a^{j}{ }_{z=} 0\right)$

$\mathrm{CS}^{\mathrm{ij}}{ }_{\mathrm{z}}=0$

Else if $\left(a^{i}{ }_{z}=X\right.$ and $\left.a_{z}^{j}=X\right)$

$\mathrm{CS}^{\mathrm{ij}}{ }_{\mathrm{z}}=\mathrm{X}$

End

End

\subsection{Example}

\begin{tabular}{|l|l|l|l|l|l|}
\hline & & T1 & & & \\
\hline & & & & S2 & \\
\hline S1 & & & & & \\
\hline & & & & & \\
\hline & & & & \\
\cline { 1 - 3 } & & T2 & & & \\
\hline
\end{tabular}

(a)

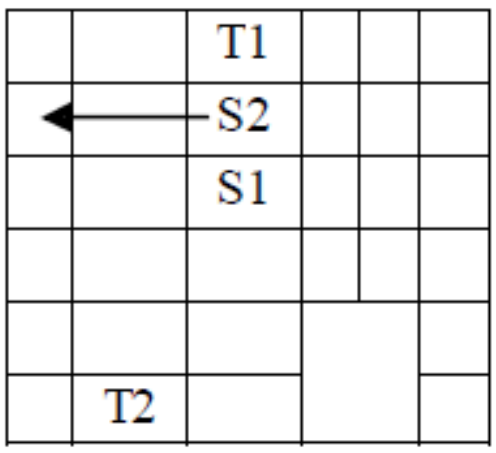

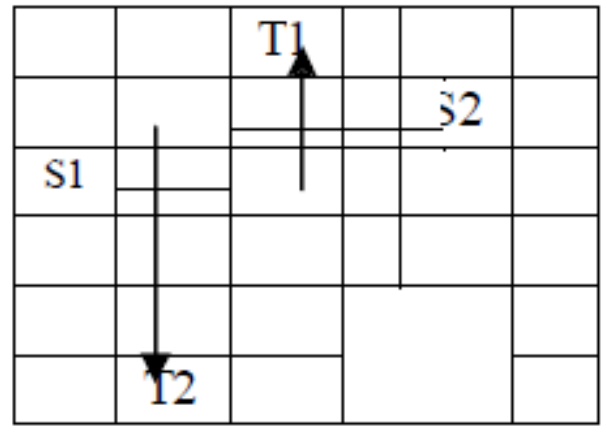

(b)

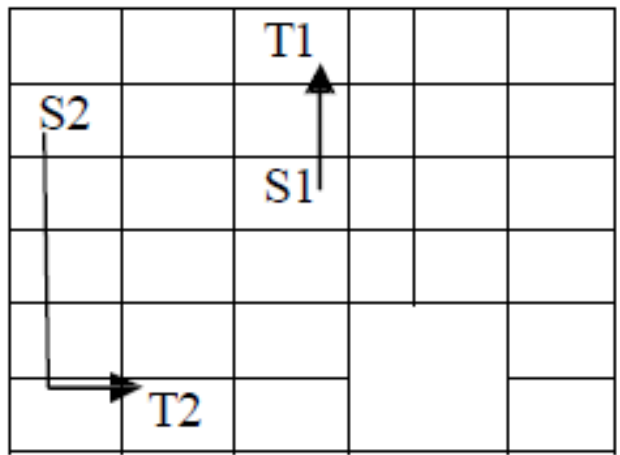

Fig. 1. Routing path of proposed method (a) A Two dimensional Array of Electrodes (b) Route path (c) Update the conflicting position in route (d) Final path

The proposed method is described in Fig. 1. Here a two dimensional grid with total thirty six electrodes with two nets and one blockage. The blockage consist of electrode $(5,4)$, $(5,5),(6,4),(6,5)$ electrodes. In figure 2(b), shows the path according to our algorithm. From these 
two net, we find the optimum path according to the position of obstacle. The fitness value of net1 is $\operatorname{Cost}(\mathrm{s} 1, \mathrm{o} 1) / \operatorname{Cost}(\mathrm{s} 1, \mathrm{t} 1)$, value is 1.625 and the fitness value of net2 $\operatorname{Cost}(\mathrm{s} 2, \mathrm{o} 1) / \operatorname{Cost}(\mathrm{s} 2, \mathrm{t} 2)$, value is 0.4 . So, net1 has lower probability to route into obstacle. The net2 has a chance, because the fitness value is less than one. But, in this problem net2 target y coordinate value is less than source y coordinate value, according to our algorithm step1, first decrease the y coordinate direction and so, avoid obstacle. If, any routing path increases the $\mathrm{x}$ coordinate direction, it would be intersect with the blockage boundary. So, our fitness value gives the correct assumption. The net1 is the global optimum because its fitness value is greater than net2. Then, we compare the routing path of net 2 with net 1 for maintaining the fluidic constraints. At timestamp2, two droplets are adjacent to each other that is the conflicting position. Droplet of net1 is at $(2,3)$ and droplet of net2 is at $(3,3)$ position at timestamp3. We update the position of net 2 droplet using equation (7), position of net2 droplet changes at $(2,1)$.

Next, we represent the graph using the routing paths, each vertex represent the electrode sequence of that time instance and each edge represents the OR sequence of adjacent vertices. Now, we find the compatible sequence of any vertex with this OR sequence and assign the same pin with any of vertex (that vertex distance from choosing vertex is greater than the other vertex) of this edge with the choosing vertex.

Here, we now find the compatible sequence between the vertexes. Let, Net1 routing path is ( $\mathrm{S}$, $\mathrm{a}, \mathrm{b}, \mathrm{c}, \mathrm{d}, \mathrm{T} 1)$ and Net 2 routing path is ( $\mathrm{S} 2, \mathrm{e}, \mathrm{f}, \mathrm{g}, \mathrm{i}, \mathrm{j}, \mathrm{k}, \mathrm{l}, \mathrm{T} 2)$. Electrode activation sequence of $\mathrm{e} 1$ and $\mathrm{e} 2$ are 10xxxxxxxx and 010xxxxxxxx respectively. The value of ' $\mathrm{a}$ ' is (e1 | e2)

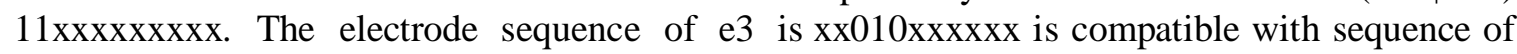
a. The value of $x$ can be replaced by 0 or 1 as per as requirement. Now, we can assign same pin at e1 and e4 because distance between e1 to e4 is greater than the distance between e 2 and e4. In this way, we can assign the pin in different electrode.

\begin{tabular}{|l|l|l|l|l|l|}
\hline & & 1 & & & \\
\hline 3 & 5 & 4 & 2 & 1 & \\
\hline 1 & 2 & 3 & & & \\
\hline & & & & & \\
\hline 5 & & & & & \\
\hline 5 & & & & & \\
\cline { 1 - 3 } 2 & 1 & & & & \\
\hline
\end{tabular}

Fig. 2. Pin assignment

In fig.2, we define the pin assignment of the cell used in routing. Here, total thirteen cells use for routing and only five pins use. So, in our method $61.5 \%$ pin reduced compare to direct addressing.

\section{RESUlTS AND DISCUSSION}

We have implemented our algorithm in Java and executed all experiment on an Intel 2.6-GHz 32-b Linux machine with 2-GB RAM.We have used In_Vitro_1, In_Vitro_2, protein_1 and protein_2 and some hard test set in our experiment. Table 1 gives the comparison for the experimental results of maximum arrival time of source droplets to the corresponding target amongst all sub-problems with some existing [3],[6],[14],[17] results. A comparative study on 
pin count reduction presents at Table2. Table3 compares with Ant Colony Optimization [12] method for cell utilization for hard test sets and gives the improved results. Table 4 represents comparison for cell utilization of the following algorithms. As, we use a multi-objective optimization

technique, all comparisons with previous method shows an improved result. Experimental results show of an improvement over the other existing methods in routing as well as pin count reduction.

Table 1. Comparison for Maximum Time and Average time per sub-problem result with $[3],[6],[14],[17]$

\begin{tabular}{|l|l|l|l|l|l|}
\hline $\begin{array}{l}\text { Benchmar } \\
\text { k (sub- } \\
\text { problems) }\end{array}$ & $\begin{array}{l}\text { Progressi } \\
\text { ve ILP[3] } \\
\text { Tmax/ } \\
\text { Tavg }\end{array}$ & $\begin{array}{l}\text { Broadcast } \\
\text { Addressi } \\
\text { ng [14] } \\
\text { Tmax } \\
/\end{array}$ & $\begin{array}{l}\text { Cross- } \\
\text { referencing } \\
{[6]} \\
\text { Tmax/ } \\
\text { Tavg }\end{array}$ & $\begin{array}{l}\text { Cross } \\
\text { router } \\
{[17]} \\
\text { Tmax/ } \\
\text { Tavg }\end{array}$ & $\begin{array}{l}\text { Our method } \\
\text { Tmax/ } \\
\text { Tavg }\end{array}$ \\
\hline $\begin{array}{l}\text { In Vitro1 } \\
(11)\end{array}$ & $\begin{array}{l}24 / \\
13.09\end{array}$ & 19.30 & 11.90 & $/ 13.55$ & $/ 10.9$ \\
\hline In & $22 /$ & $17 /$ & 17 & $19 /$ & 17 \\
Vitro & 11.00 & 11.01 & $/ 10.26$ & 10.73 & $/ 11.02$ \\
\hline $\begin{array}{l}\text { Protein } \\
1(64)\end{array}$ & $26 /$ & $20 /$ & $20 /$ & 20 & 20 \\
\hline $\begin{array}{c}\text { Protein } \\
2(78)\end{array}$ & $26 / 15$ & 16.55 & 15.18 & $/ 15.52$ & $/ 15.07$ \\
\hline
\end{tabular}

Table 2. Comparison for Pin count reduction

\begin{tabular}{|c|c|c|c|c|c|}
\hline Benchmark & $\begin{array}{l}\text { Direct } \\
\text { Addressing } \\
\text { Pin max / } \\
\text { Pin avg }\end{array}$ & $\begin{array}{l}\text { Broadcast } \\
\text { Addressing } \\
\text { Pin max / } \\
\text { Pin avg }\end{array}$ & $\begin{array}{l}\text { Cross } \\
\text { referencing } \\
\text { Pin max / } \\
\text { Pinavg }\end{array}$ & $\begin{array}{l}\text { Two } \\
\text { stage } \\
\text { ILP } \\
\text { Pin } \\
\text { max/ } \\
\text { Pinavg }\end{array}$ & $\begin{array}{l}\text { Our } \\
\text { method } \\
\text { Pin max } \\
\text { / } \\
\text { Pinavg }\end{array}$ \\
\hline In Vitro 1 & $\begin{array}{l}45 / \\
21.55\end{array}$ & \begin{tabular}{|l|}
$21 /$ \\
9.48
\end{tabular} & $\begin{array}{c}25 / \\
14.55\end{array}$ & $\begin{array}{l}18 / \\
9.49\end{array}$ & $\begin{array}{l}21 / \\
9.45\end{array}$ \\
\hline In Vitro2 & $\begin{array}{l}42 / \\
15.73\end{array}$ & \begin{tabular}{|l|}
$21 /$ \\
8.95
\end{tabular} & $\begin{array}{c}24 / \\
12.76\end{array}$ & $\begin{array}{l}17 / \\
9.21\end{array}$ & $\begin{array}{l}16 / \\
8.78\end{array}$ \\
\hline
\end{tabular}


International Journal on Information Theory (IJIT), Vol.3, No.1, January 2014

Table 3. Comparison for total cell utilization with [3],[6],[14],[17]

\begin{tabular}{|c|l|c|c|c|c|}
\hline $\begin{array}{c}\text { Benchmark } \\
\text { (sub- } \\
\text { problems) }\end{array}$ & $\begin{array}{l}\text { Progressive } \\
\begin{array}{l}\text { ILP[3] } \\
\text { (cell } \\
\text { Utilization) }\end{array}\end{array}$ & $\begin{array}{l}\text { Broadcast } \\
\text { Addressing } \\
{[14]} \\
\text { (cell } \\
\text { Utilizati } \\
\text { on) }\end{array}$ & $\begin{array}{l}\text { Cross } \\
\text { referencing } \\
{[6]} \\
\text { (cell } \\
\text { Utilizati } \\
\text { on) }\end{array}$ & $\begin{array}{c}\text { Cross } \\
\text { router } \\
{[17]} \\
\text { (cell } \\
\text { Utilizatio } \\
\text { n) }\end{array}$ & $\begin{array}{c}\text { Our } \\
\text { method (cell } \\
\text { Utilization }\end{array}$ \\
In Vitro 1 (11) & 231 & 237 & 223 & 246 & 212 \\
\hline $\begin{array}{l}\text { In Vitro2 } \\
(15)\end{array}$ & 231 & 236 & 226 & 250 & 192 \\
\hline $\begin{array}{l}\text { Protein 1 } \\
\text { (64) }\end{array}$ & 1597 & 1618 & 1521 & 1664 & 1516 \\
\hline $\begin{array}{l}\text { Protein 2 } \\
(78)\end{array}$ & 927 & 939 & 919 & 952 & 923 \\
\hline
\end{tabular}

Table 4. Comparison of our method with [12] for Random Test instances

\begin{tabular}{|c|c|c|c|c|}
\hline $\begin{array}{c}\text { Bench Mark } \\
\text { (sub- } \\
\text { problems) }\end{array}$ & $\begin{array}{c}\text { Unit cell } \\
\text { used [12] }\end{array}$ & $\begin{array}{c}\text { Completion } \\
\text { Time[12] }\end{array}$ & $\begin{array}{l}\text { Unit cell } \\
\text { used(our } \\
\text { method) }\end{array}$ & $\begin{array}{l}\text { Completion } \\
\text { Time( our } \\
\text { method) }\end{array}$ \\
\hline test_1 & 81 & 23 & 76 & 35 \\
\hline test_5 & 143 & 30 & 121 & 28 \\
\hline test_11 & 321 & 41 & 280 & 42 \\
\hline test_17 & 573 & 52 & 445 & 47 \\
\hline
\end{tabular}

\section{Conclusion}

This paper is described the new optimized routing algorithm using evolutionary algorithm concept. In this paper, the fitness function is designed in such way that algorithm is given an optimized result. The routing algorithm is executed multiple route concurrently and it is one of the main time-consuming phase of the algorithm. A masked based addressing method is also proposed using the routing result for pin constraint design of digital micro-fluidic biochips. The algorithm is shown how easily compatible sequences are identified. The reduction of number of pins may lead to electrode - interference but the proposed method is solved it. The timecomplexity of the algorithm is bound into polynomial time.

This paper is proposed the routing method and pin-assignment only for 2D micro fluidic biochip. The work can be extended for 3D micro fluidic biochip. The proper designing and scheduling of the bioassay is the big challenge for the 3D biochip. As it is used in safety critical application, testing in pin-constraint micro fluidic biochip is vast era in this field. 
International Journal on Information Theory (IJIT), Vol.3, No.1, January 2014

\section{REFERENCES}

[1] rinvasan, V., Pamula, V. K., Pollack, M. G., Fair, R. B.: Clinical diagnostics on human whole blood, plasma, serum, urine, saliva, sweat, and tears on a digital microfluidic platform, Proceeding of Miniaturized Systems for Chemistry pp. 1287-1290, (2003)

[2] Guiseppi-Elie, A., Brahim, S., Slaughter, G., Ward, K. R.: Design of a sub cut a- neous implantable biochip for monitoring of glucose and lactate, IEEE Sensors Journal, vol. 5, no. 3, pp. 345-355, (2005)

[3] P. Yuh, S. Sapatnekar, C. Yang, Y. Chang : A progressive-ilp base routing algorithm for cross referencing biochips," Design Automation Conference, pp. 284-289, (2008)

[4] Böhringer K. F. :Modeling and controlling parallel tasks in droplet based microfluidic systems," IEEE Trans. Comput.-Aided Design Integr. Circuits Syst., vol. 25, no. 2, pp. 334-344, Feb. (2006)

[5] Griffith E. J., Akella S., Goldberg M. K. : Performance characterization of a reconfigurable planararray digital microfluidic system," IEEE Trans. Comput.-Aided Design Integr. Circuits Syst., vol. 25, no. 2, pp. 345-357, Feb. (2006)

[6] V. Pamula V. Srinivasan ,R. Fair : An integrated digital microfluidic lab-on-a-chip for clinical diagnostics on human physiological fluids. Journal of Lab Chip, 4:310 - 315, (2004)

[7] T. W. Huang ,T. Y. Ho. :A fast routability and performance driven droplet routing algorithm for digital microfluidic biochips." In Proceeding of IEEE International Conference on Computer Design, page 445450 , (2009)

[8] T. Xu , K. Chakrabarty.: Integrated droplet routing and defect tolerance in the synthesis of digital microfluidic biochips. ACM Journal on Emerging Technologies in Computing Systems,4(3:II):11.1 $11.24,(2008)$

[9] Zigang Xiao ,Evangeline F. Y.Young. : Droplet-routing-aware module placement for crossreferencing biochips. In Proceedings of International Symposium on Physical Design, pages193 199. California, USA, (2010)

[10] Chia-Lin Yang Ping-Hung Yuh and Yao-Wen Chang.: A network-flow based routing algorithm for digital microfluidic biochips. In Proceedings of IEEE/ACM Int. Conf. on Computer-Aided Design, pages $752-757,(2007)$

[11] T. W. Huang, T. Y. Ho. : A fast routability and performance driven droplet routing algorithm for digital microfluidic biochips. In Proceeding of IEEE International Conference on Computer Design, page 445 450,(2009)

[12] Indrajit Pan, Parthasarathi Dasgupta, Hafizur Rahaman, Tuhina Samanta: Ant Colony Optimization Based Droplet Routing Technique in Digital Microfluidic Biochip Ant Colony, International Symposium on Electronic System Design, page 224-228,(2011)

[13] C. H. Lin T. W. Huang and T. Y. Ho.: A contamination aware droplet routing algorithm for the synthesis of digital microfluidic biochips. In IEEE Transaction on Computer Aided Design of Integrated Circuits and Systems, page 1682 1695, (2010)

[14] Tao Xu , Krishnendu Chakrabarty,: Broadcast Electrode-Addressing for Pin-Constrained MultiFunctional Digital Microfluidic Biochips, Design Automation Conference, June, Anaheim, California USA, pp 173 - 178 (2009)

[15] T. $\mathrm{Xu}, \mathrm{K}$. Chakrabarty: Droplet-trace-based array partitioning and a pin assignment algorithm for the automated design of digital microfluidic biochips, IEEE/ACM CODES+ISSS, pp. 112-117, (2006)

[16] S. K. Fan et al: Manipulation of multiple droplets on $\mathrm{N} \times \mathrm{M}$ grid by cross-reference EWOD driving scheme and pressure-contact packaging, Proc. IEEE MEMS Conf., pp. 694-697, (2003)

[17] Zigang Xiao, Evangeline F.Young: Cross Router, a droplet router for cross-referencing digital micro fluidic biochips, ASPDAC '12 Proceedings of the 2012 Asia and South Pacific Design Automation Conference, Taipei, Taiwan,, pp 187-192(2012) 